\title{
Tailoring the valve choice to the patient: contemporary choices and their optimal applications
}

\author{
Ben Wilkins, Lars Søndergaard, Ole De Backer \\ The Heart Center, Rigshospitalet Copenhagen University, Copenhagen, Denmark \\ Correspondence to: Ole De Backer, MD, PhD. Consultant Interventional Cardiology, The Heart Center, Rigshospitalet Copenhagen University, \\ Blegdamsvej 9, 2100 Copenhagen, Denmark. Email: ole.debacker@gmail.com.
}

Submitted Mar 08, 2020. Accepted for publication Apr 28, 2020.

doi: 10.21037/acs-2020-av-14

View this article at: http://dx.doi.org/10.21037/acs-2020-av-14

\section{Clinical vignette}

In recent years, treatment options for patients with severe symptomatic aortic valve stenosis (AS) have expanded significantly. Previously restricted to extreme and high surgical risk profile patients, there is now evidence for the use of transcatheter aortic valve replacement (TAVR) in elderly patients at intermediate and low surgical risk. Along with this expanded patient population comes an expanded set of individual patient characteristics that warrant careful consideration when selecting the optimal transcatheter heart valve (THV) system. The numerous TAVR systems currently available function well in a wide range of patients, but have key differences in vascular access options, insertion profile, delivery system flexibility, treatable annulus range, THV expansion mechanics, leaflet position, likelihood of inducing conduction abnormalities, etc. No single system has outright superior performance characteristics. A detailed understanding of individual THV system strengths and weaknesses is important in optimizing clinical TAVR outcomes.

\section{Procedural techniques}

Meticulous preprocedural planning is paramount for an optimal TAVR outcome. The importance of highresolution computed tomography (CT) imaging of the aortic valve complex and the access vessels-and its careful interpretation—must be emphasized.

\section{Access}

Considerations for TAVR system selection relate frequently to vascular access. It is commonplace to encounter severe iliofemoral and aortic vessel disease in TAVR candidates (1), making the delivery system insertion profile and flexibility highly relevant or driving the selection of an alternative vascular access site. In general, an iliofemoral vessel diameter of $\geq 5 \mathrm{~mm}$ is acceptable for transfemoral TAVRalthough tortuosity, calcifications and atheroma must be taken into consideration. Devices with smaller insertion profiles are available and may include an integrated sheath on the delivery system. Additionally, several manufacturers have released low-profile expandable introducer sheaths, designed to radially dilate as the THV passes through the iliofemoral artery, but thereby also reducing longitudinal forces on the access vessel.

In cases of unsuitable transfemoral access, the alternative access may require a low profile and flexible TAVR system negotiating tortuous axillary, subclavian or carotid vasculature. This minimizes the insertion profile for transcaval crossing, or may require transapical or direct aortic delivery of the transcatheter aortic bioprosthesis.

\section{Aortic valve and aortic root}

Dimensions from the aortic annulus and aortic root generally dictate the choice of the THV size. Within this guide, however, there is significant room for variation in device selection. Large annulus measurements (perimeter $>85 \mathrm{~mm}$, area $>573 \mathrm{~mm}^{2}$ ) are within the treatable range of only two currently available THV systems $(29 \mathrm{~mm}$ Sapien 3, Edwards Lifesciences, CA, USA, and $34 \mathrm{~mm}$ Evolut $\mathrm{R}$, Medtronic, MN, USA), although large valve sizes are also in development for most other THV systems. Small 
aortic annulus size or a valve-in-valve procedure (treating a surgical aortic bioprosthesis with a small true inner diameter) are optimally treated with a self-expanding THV with supra-annular leaflet position. This maximizes the aortic valve opening area and reduces transvalvular gradients, which is likely to be important to improve THV longevity (2).

In cases of a severely calcified left ventricular outflow tract and/or sinotubular junction with small dimensions, it is best to avoid a balloon expandable THV given the potential risk for annulus rupture and aortic dissection. A horizontal aorta, defined as an aortic angulation $>60^{\circ}$, suits a flexible or steerable delivery system, ensuring proper alignment of the TAVR system with the native aortic annulus and thereby simplifying THV positioning. Low coronary ostia or a high likelihood of future percutaneous coronary intervention favors the use of a THV with low stent frame and/or intra-annular leaflet position, thereby allowing a less obstructed coronary access. A heavily or asymmetrically calcified annulus and left ventricular outflow tract should be managed with TAVR systems with an outer sealing skirt to reduce the risk of paravalvular leak (PVL) - although not all types of sealing materials seem to be effective. The risk for PVL can be further minimized by the presence of a large-cell THV stent frame design, or a mechanically expandable stent frame allowing for close annulus apposition. Consideration should also be given to avoid THV oversizing and that THVs with lower radial force may be less inclined to induce atrioventricular (AV) conduction disturbance. However, it is important to realize that the choice of THV type, THV size and implantation depth is a complex interplay that will determine the final risk of PVL and conduction disturbance.

Although early results from large registries show good performance of THV in bicuspid patients with newer generation devices (3), it is important to recognize that patients with bicuspid anatomy have been excluded from the large randomized trials that have introduced TAVR into mainstream practice. As a result, treatment of bicuspid AS remains an off-label indication for TAVR. Careful valve and size selection to account for asymmetric valve constriction and the greater risk for PVL, annulus rupture, and pacemaker-as well as the use of cerebral embolic protection-will likely optimize TAVR results in this specific patient population.

Similarly, pure non-calcified native aortic valve regurgitation (NAVR) has been treated via TAVR in off-label use. However, referral to surgery is the most appropriate initial consideration in this setting due to unpredictable anchoring of the THV. One device, the JenaValve (JenaValve Technologies Inc., CA, USA), was designed with 'clips' that should make it amenable to treat non-calcified NAVR and is currently under investigation in a clinical study.

\section{Patient characteristics}

Physiological patient characteristics may play a key role in TAVR customization as well. Patients with pre-existing conduction disturbance, but no permanent pacemaker, should be considered for high implantation of a balloon expandable THV, given trial evidence of low pacemaker rates, albeit in a highly selected patient cohort (4). Alternatively, they can be considered for a self-expandable THV with lower radial force (5), which may minimize trauma to the membranous septum and avoid the induction of a bundle brunch block or high-degree AV block. Intraprocedurally, patients with severe left ventricular dysfunction are more likely to remain hemodynamically stable if rapid ventricular pacing is not required and if the THV functions early in deployment.

Finally, the expansion of TAVR to patients with longer life expectancy also means that the overall patient lifecycle should be taken into consideration individually. Not only will early device safety and performance be a key determinant of success, but valve durability, long-term effects of conduction disturbance, and coronary access will also be important aspects to consider. Comparative studies investigating the impact of THV choice on these issues demands longer-term follow-up than the current head-tohead comparative THV studies with generally short followup times.

\section{Comments}

TAVR is an evolving intervention, with a growing body of evidence to support its expanding use. There are several head-to-head comparative THV studies comparing different THV types, e.g., CHOICE, REPRISE, PORTICO-IDE and SCOPE-I/II clinical trials. These studies have generated data on early THV performance; however, the act of patient randomization fails to consider the noted differences in THV technology that may suit some patient and anatomic characteristics more than others. Daily clinical TAVR practice has already moved towards optimizing individual patient results through a 
process of patient-tailored TAVR. A detailed knowledge of contemporary TAVR technology and its applications has expanded the treatable TAVR population and is likely to further improve patient outcomes.

\section{Acknowledgments}

Funding: None.

\section{Footnote}

Conflicts of Interest: The authors have no conflicts of interest to declare.

Open Access Statement: This is an Open Access article distributed in accordance with the Creative Commons Attribution-NonCommercial-NoDerivs 4.0 International License (CC BY-NC-ND 4.0), which permits the noncommercial replication and distribution of the article with the strict proviso that no changes or edits are made and the original work is properly cited (including links to both the formal publication through the relevant DOI and the license). See: https://creativecommons.org/licenses/by-nc-nd/4.0/.

Cite this article as: Wilkins B, Søndergaard L, De Backer O. Tailoring the valve choice to the patient: contemporary choices and their optimal applications. Ann Cardiothorac Surg 2020;9(6):522-524. doi: 10.21037/acs-2020-av-14

\section{References}

1. Mohananey D, Villablanca P, Gupta T, et al. Association of peripheral artery disease with in-hospital outcomes after endovascular transcatheter aortic valve replacement. Catheter Cardiovasc Interv 2019;94:249-55.

2. Sondergaard L, Ihlemann N, Capodanno D, et al. Durability of Transcatheter and Surgical Bioprosthetic Aortic Valves in Patients at Lower Surgical Risk. J Am Coll Cardiol 2019;73:546-53.

3. Yoon SH, Bleiziffer S, De Backer O, et al. Outcomes in Transcatheter Aortic Valve Replacement for Bicuspid Versus Tricuspid Aortic Valve Stenosis. J Am Coll Cardiol 2017;69:2579-89.

4. Mack MJ, Leon MB, Thourani VH, et al. Transcatheter Aortic-Valve Replacement with a Balloon-Expandable Valve in Low-Risk Patients. N Engl J Med 2019;380:1695-705.

5. Toggweiler S, Nissen H, Mogensen B, et al. Very low pacemaker rate following ACURATE neo transcatheter heart valve implantation. EuroIntervention 2017;13:1273-80. 